\title{
The continued hunt for the elusive standard short regimen for treatment of multidrug-resistant tuberculosis
}

\author{
J. Peter Cegielski ${ }^{1}$, Payam Nahid $\mathbb{1}^{2}$ and Giovanni Sotgiu $\mathbb{1}^{3}$ \\ Affiliations: ${ }^{1}$ Dept of Epidemiology, Rollins School of Public Health, Emory University, Atlanta, GA, USA. \\ ${ }^{2}$ School of Medicine, University of California at San Francisco, UCSF Center for Tuberculosis, San Francisco, \\ CA, USA. ${ }^{3}$ Dept of Medical, Surgical and Experimental Sciences, University of Sassari, Sassari, Italy.
}

Correspondence: J. Peter Cegielski, 1361 Council Bluff Drive NE, Atlanta, GA 30345, USA. E-mail: peter. cegielskidgmail.com

@ERSpublications

In low- and middle-income countries, treatment of highly selected MDR-TB patients with a standardised 9-12 month regimen leads to $\mathbf{8 0 \%}$ or better successful treatment outcomes http://bit.ly/384SFpY

Cite this article as: Cegielski JP, Nahid P, Sotgiu G. The continued hunt for the elusive standard short regimen for treatment of multidrug-resistant tuberculosis. Eur Respir J 2020; 55: 2000224 [https://doi.org/ 10.1183/13993003.00224-2020].

With three new and three recently repurposed drugs, treatment of multidrug-resistant tuberculosis (MDR-TB) is more hopeful today than at any time since it emerged in the 1980s and 1990s [1-3]. This optimism contrasts starkly with dismal global treatment success rates of $48-56 \%$ as reported by the World Health Organization (WHO) in its annual Global Tuberculosis Reports from 2014 to 2019 [4, 5].

Before 2010, treatment consisted of four to five drugs given for about 24 months, including daily injections for the first 6 to 8 months. In 2010, VAN DEUN et al. [6] challenged this paradigm based on their experience in Bangladesh. With stepwise improvements in successive cohorts, they arrived at a more intensive regimen curing $\sim 80 \%$ of individuals in $9-12$ months. The regimen included seven drugs with daily injections for the first 4 to 5 months, followed by four to five drugs for the remainder. At first, the results were plausible because the principle that "more drugs are better" had deep historical roots in TB chemotherapy. Subsequently, these results were replicated in observational studies in many countries $[7,8]$, followed by the first-ever full-length, multinational randomised controlled treatment trial for MDR-TB (STREAM Stage 1, ClinicalTrials.gov NCT02409290), comparing the WHO-recommended standardised shorter regimen with standardised longer regimens based on WHO recommendations in effect at that time $[9,10]$. In every case, the results show approximately $\geqslant 80 \%$ success rates against MDR-TB caused by Mycobacterium tuberculosis strains without known resistance to the drugs in the regimen (with the only exception of isoniazid), similar to outcomes with conventional longer regimens.

In this issue of the European Respiratory Journal, ABIDI et al. [11] extend this knowledge base by reporting the results of an individual patient data meta-analysis comparing the standardised shorter regimen with individualised longer regimens. However, a key issue to consider is that individualised longer regimens, considered to be the standard of care in high-income countries, have remained out of reach of the majority of MDR-TB patients who live in low and middle-income countries and this is reflected in the datasets analysed by ABIDI et al. [11]. Consequently, it is important to acknowledge that even though epidemiological, demographic, clinical and microbiological differences may be controlled with

A typographical error has been corrected in this article, which was republished on 7 April, 2020.

Received: 02 Feb 2020 | Accepted after revision: 03 Feb 2020

Copyright CERS 2020 
state-of-the-art statistical methods, the programmes and countries using longer versus shorter regimens differed almost categorically, and cannot be statistically adjusted. Still, achieving $80 \%$ success (as good as the standard of care in high-income countries) is an important affirmation of this quantum step forward in global efforts to control this pandemic. The fly in the soup is that this shorter regimen, standardised according to WHO guidelines, only performs at this level of success when there is no additional drug resistance, thereby applying to only one quarter to one-third of MDR-TB patients in most countries [12]. ABIDI et al. [11] showed that it makes no sense to use a drug to which the patient's isolate is resistant because failure/relapse or death were consistently higher in patients whose isolates had resistance to one or more of the drugs.

Standardised regimens can certainly be modified, but then they would no longer be standardised. The temptation to switch out kanamycin, for example, is understandable and justified, but this approach has both positive and negative implications. All of the published data to date describe the standardised regimen without modifications, and the implications for efficacy, safety and tolerability of modifications remains unknown. At the same time, not substituting weak drugs with newer drugs known to be better is illogical. The latest evidence from individual patient data meta-analyses of 50 observational studies published since 2009 indicates that kanamycin and capreomycin are inferior and should be replaced by amikacin (or streptomycin, if susceptible) or, even better, by oral agents such as bedaquiline [13, 14]. Indeed, at least four of the seven drugs in the shorter regimen should be reconsidered. High-dose isoniazid in patients with isoniazid-resistant TB, while based on first principles, is not based on evidence and its effect is limited to a minority of cases at best, specifically those with low-level resistance. The use of ethambutol and pyrazinamide in patients with $\geqslant 50 \%$ prevalence of resistance would not be considered good medical practice in circumstances where timely drug susceptibility testing is available for those drugs [12]. The standardised shorter regimen is already outdated. Consequently, WHO has recently issued guidance, not based on direct evidence, allowing specific substitutions under these circumstances [15]

Likewise, individualised longer regimens described in the study by ABIDI et al. [11] are now outdated as well. First, results of an overlapping but much larger individual patient data meta-analysis that informed the 2018 WHO and 2019 American Thoracic Society/European Respiratory Society/Infectious Diseases Society of America/US Centers for Disease Control and Prevention guidelines promoted bedaquiline and linezolid to first-choice drugs, while demoting older, more toxic or less effective drugs (aminoglycosides, thionamides, PAS) $[13,14,16]$. Post-hoc analysis of those data by ABIDI et al. [11], which carved out a small group of subjects whose treatment conformed to these new guidelines, begins to address that gap. Secondly, longer regimens may become obsolete based on reports from South Africa describing 6-month regimens of three or four new/repurposed drugs, in some cases combined with a fluoroquinolone or pyrazinamide, which improved treatment outcomes for patients with extensively drug-resistant TB from $\sim 20 \%$ to $\sim 80 \%$ [17-20]. This work led to the approval of pretomanid, the third new drug approved for MDR-TB in the past 7 years $[21,22]$.

Several new (bedaquiline, delamanid and pretomanid) and repurposed (linezolid, clofazimine and carbapenems) oral agents appear to be effective and safe enough to use in a majority of MDR-TB patients. Although these developments are promising, policy guidance rests largely on observational cohort data rather than clinical trials. Now we urgently need new clinical trials that would identify an effective shorter regimen because that would reduce the duration, cost, complexity and delays for all future clinical trials on MDR-TB. Rather than comparing every new regimen against a 2 -year regimen, the comparison group can and should become a 6- or 9-month regimen, shortening the task of clinical trials to the same duration as drug-susceptible TB. With good enough drugs, we know TB can be cured with 6 months' of treatment. We must identify, then deploy drugs that are at least as effective as that, circumventing known mechanisms of resistance. With the advent of newer agents and all-oral shortened duration regimens for MDR-TB, current policy decisions making outsized reliance on observational cohort and TB registry data for developing practice guidelines should be reconsidered and investment into the conduct of TB clinical trials should be intensified.

Whereas well-performed meta-analyses of observational research can produce valid, compelling results [13, 23], observational data are low quality because of the inordinate risk of unknown or unmeasured confounding, often leading to significant heterogeneity between studies [13, 23]. While individual patient data meta-analysis offers important evidence in the absence of clinical trials, they are not optimal and ought not be the sole basis for policy decisions and treatment recommendations as new regimens emerge. Finally, meta-analyses are only as good as the data incorporated into them. Without standardisation/ quality control of data collection with close monitoring and long-term follow-up of patients, the certainty in the evidence and outcomes of critical importance is low.

TB patients (and TB programmes, providers and policy decision makers) deserve the highest quality evidence-base that can be generated by randomised controlled clinical trials. The US Agency for 
International Development, the Global Alliance, the US Centers for Disease Control and Prevention and UNITAID are spear-heading high-quality treatment trials in TB (www.newtbdrugs.org/pipeline/developers, www.newtbdrugs.org/pipeline/clinical). The US National Institutes of Health moved in that direction with the RePORT network, starting with prospective observational research to develop international capacity for clinical trials. The international Working Group on New TB Drugs reports several planned or ongoing phase 1 and phase 2 trials of newer and repurposed drugs (www.newtbdrugs.org/pipeline/trials), therein lies the hope. In the meantime, most MDR-TB patients, especially in low and middle-income countries, continue to be treated with kanamycin, ofloxacin, thionamides and other older, weaker drugs; therein lies the dissatisfaction with the status quo.

Conflict of interest: J.P. Cegielski has nothing to disclose. P. Nahid has nothing to disclose. G. Sotgiu has nothing to disclose.

\section{References}

1 Stottmeier KD. Emergence of rifampin-resistant Mycobacterium tuberculosis in Massachusetts. J Infect Dis 1976; 133: 88-90.

2 Centers for Disease Control. Meeting the challenge of multidrug-resistant tuberculosis. MMWR Recomm Rep 1992; 41: 49-57.

3 World Health Organization (WHO)/International Union Against Tuberculosis and Lung Disease Global Project on Drug Resistance Surveillance. Anti-Tuberculosis Drug Resistance in the World 1997. Geneva, World Health Organization, 1997.

4 World Health Organization. Global Tuberculosis Report 2014. Geneva, World Health Organization, 2014.

5 World Health Organization. Global Tuberculosis Report 2019. Geneva, World Health Organization, 2019.

6 Van Deun A, Maug AK, Salim MA, et al. Short, highly effective, and inexpensive standardized treatment of multidrug-resistant tuberculosis. Am J Respir Crit Care Med 2010; 182: 684-692.

7 Trébucq A, Decroo T, Van Deun A, et al. Short-course regimen for multidrug-resistant tuberculosis: a decade of evidence. J Clin Med 2019; 9: E55.

8 Trébucq A, Schwoebel V, Kashongwe Z, et al. Treatment outcome with a short multidrug-resistant tuberculosis regimen in nine African countries. Int J Tuberc Lung Dis 2018; 22: 17-25.

9 Moodley R, Godec TR, STREAM Trial Team. Short-course treatment for multidrug-resistant tuberculosis: the STREAM trials. Eur Respir Rev 2016; 25: 29-35.

10 Nunn AJ, Phillips PPJ, Meredith SK, et al. A trial of a shorter regimen for rifampin-resistant tuberculosis. $N$ Engl $J$ Med 2019; 380: 1201-1213.

11 Abidi S, Achar J, Neino MMA, et al. Standardised shorter regimens versus individualised longer regimens for rifamoin- or multidrug-resistant tuberculosis. Eur Respir J 2020: 55: 1901467.

12 Dalton T, Cegielski P, Akksilp S, et al. Prevalence of and risk factors for resistance to second-line drugs in people with multidrug-resistant tuberculosis in eight countries: a prospective cohort study. Lancet 2012; 380: 1406-1417.

13 Collaborative Group for the Meta-Analysis of Individual Patient Data in MDR-TB treatment-2017, Ahmad N, Ahuja SD, et al. Treatment correlates of successful outcomes in pulmonary multidrug-resistant tuberculosis: an individual patient data meta-analysis. Lancet 2018; 392: 821-834.

14 Nahid P, Mase SR, Migliori GB, et al. Treatment of drug-resistant tuberculosis. an official ATS/CDC/ERS/IDSA clinical practice guideline. Am J Respir Crit Care Med 2019; 200: e93-e142.

15 World Health Organization. Rapid communication: key changes to treatment of drug-resistant tuberculosis. Geneva, World Health Organization, 2019.

16 World Health Organization. WHO consolidated guidelines on drug-resistant tuberculosis treatment. Geneva, World Health Organization, 2019.

17 Conradie A, Everitt D, Mendel C, et al. Sustained high rate of successful treatment outcomes: interim results of 75 patients in the Nix-TB clinical study of pretomanid, bedaquiline and linezolid. Int J Tuberc Lung Dis 2018; 22: Suppl. 2, S69.

18 Dawson R, Diacon AH, Everitt D, et al. Efficiency and safety of the combination of moxifloxacin, pretomanid (PA-824), and pyrazinamide during the first 8 weeks of antituberculosis treatment: a phase $2 \mathrm{~b}$, open-label, partly randomised trial in patients with drug-susceptible or drug-resistant pulmonary tuberculosis. Lancet 2015; 385: 1738-1747.

19 Evaluation of Early Bactericidal Activity in Pulmonary Tuberculosis With Clofazimine (C)-TMC207 (J)-PA-824 (Pa)-Pyrazinamide (Z) (NC-003). Identifier: NCT01691534. ClinicalTrials.gov Date last updated: 13 December 2016; date last accessed: 25 January 2020.

20 Conradie F, Diacon AH, Everitt D, et al. The NiX-TB trial of pretomanid, bedaquiline and linezolid to treat XDR-TB. Abstract 80LB. www.croiconference.org/sessions/nix-tb-trial-pretomanid-bedaquiline-and-linezolid-treat$\mathrm{xdr}-\mathrm{tb}$

21 US Food and Drug Administration. www.fda.gov/news-events/press-announcements/fda-approves-new-drugtreatment-resistant-forms-tuberculosis-affects-lungs Date last updated: 14 August 2019; date last accessed: 25 January 2020.

22 Keam SJ. Pretomanid: first approval. Drugs 2019; 79: 1797-1803.

23 Fox GJ, Benedetti A, Mitnick CD, et al. Propensity score-based approaches to confounding by indication in individual patient data meta-analysis: non-standardized treatment for multidrug resistant tuberculosis. PLoS One 2016; 11: e0151724. 